\title{
Screening of Burns Unit Staff of a Tertiary Care Hospital for Methicillin-Resistant Staphylococcus Aureus Colonisation
}

\author{
Preetha Aravind, M.B.B.S., Prabha Unny Krishnan ${ }^{\dagger}$, M.D., D.N.B., \\ Dip.R.C.Path., Hiresave Srinivasa ${ }^{* \dagger}$, M.D. and Vijay Joseph ${ }^{\S}$, M.S., M.C.H.
}

\begin{abstract}
Staphylococcus aureus is a significant nosocomial pathogen and the development of resistance to methicillin poses a major threat to its control. This study was conducted over a three month period in a Burns Unit of a tertiary care hospital to determine the prevalance of methicillinresistant $S$. aureus (MRSA) colonisation in health care workers. All health care workers were screened using swabs from the hairline, nostril, axilla, and hands. Seventeen of $\mathbf{3 4}$ health care workers screened were MRSA-positive; 16 people tested positive for the methicillin-sensitive strain of $S$. aureus, 7 of whom were also MRSA-positive at a different site. In total, over two thirds of all health care workers were colonised by $S$. aureus. Pus samples from patients admitted in the same unit over the three month study period were analysed and showed that $21 \%$ of patients were infected or colonised with MRSA. Although a direct causal relationship is not established by these data, it is reasonable to assume that transmission from colonised health care workers is responsible, at least in part, for the extent of infection/colonisation among patients. These findings identify the need for a well defined policy for screening health care workers and controlling the rates of colonisation with potentially dangerous pathogens given the risk of transmission to susceptible patients.
\end{abstract}

\section{INTRODUCTON}

Staphylococcus aureus is one of the most versatile nosocomial (i.e. acquired in hospital) pathogens $(1,2)$. The widespread use of penicillin in the 1950's saw the spread of penicillin resistant $S$. aureus in hospitals, after which time methicillin and its derivatives became the drugs of choice for the treatment of infections caused by this organism $(3,4)$. Even before methicillin was widely used, a strain of $S$. aureus with natural resistance to this antibiotic was identified by Jevons in 1961 (5).

\footnotetext{
* To whom correspondence should be addressed: Department of Microbiology, St. John's Medical College, Bangalore, 560 034, India. $\dagger$ Department of Microbiology, St. John's Medical College \& Hospital. $\S$ Department of Plastic Surgery, St. John's Medical College \& Hospital.
}

Thereafter, methicillin-resistant $S$. aureus (MRSA) emerged as a major pathogen world-wide (6-11). Several surveys have confirmed that the incidence of MRSA varies by region; during the last 20 years, the proportion of isolates resistant to methicillin has ranged from less than $1 \%$ in Scandinavia to more than $30 \%$ in Spain, France, Italy and India (12-14).

In recent years, MRSA has become a particularly significant problem in Indian hospitals (15). In one study conducted in a tertiary care hospital in India, MRSA carriage rate ranged between $28.4 \%$ in outpatients to $33.5 \%$ in in-patients (16). MRSA is often seen in tertiary care hospitals with special care units such as burns, surgical, pulmonary and trauma units (17). The burns unit is a particularly fertile environment for MRSA because of open wounds, frequent dressing 
changes requiring handling by multiple health care workers (HCW), use of intraluminal devices, and the inherent immuno-compromised state of burn patients (17-19).

MRSA is usually introduced into an institution by a colonised (i.e. harbouring the pathogen but without discernable signs of disease) or infected patient or HCW (20). It is well known that colonisation with MRSA precedes infection (21). Several modes of transmission exist, including transient colonisation of hospital staff and contact with heavily contaminated fomites and environmental surfaces around infected patients $(7,22)$. Factors contributing to transmission and perpetuation of this organism include prolonged hospital stay and the use of several broad spectrum antimicrobial agents $(14,23)$. Carriage of the pathogen by HCWs who can transmit the pathogen to the patients with whom they have contact also directly contributes to the continuance of the problem $(21,24)$.

At St. John's Medical College Hospital (SJMCH), although MRSA is frequently isolated and reported from the Burns Unit in random screening procedures, there is no well documented data on the carriage rate of this pathogen among HCWs. An earlier study had shown that infection with MRSA in this specialty had reached high proportion (25). The aim of this study was to assess the carriage rate of MRSA among HCWs in the Burns Unit. In order to illustrate the possible implications of the high carriage rate among $\mathrm{HCWs}$, an attempt was also made to estimate the number of patients colonised or infected by the same organism in this unit during the study period.

\section{MATERIALS \& METHODS}

\section{Staff screening}

The study period extended from June to August, 1997. All 34 staff members working in the Burns Unit including doctors, nurses and other workers who had access to the patients' rooms were screened for $S$. aureus. Informed consent was obtained prior to the screening. Swabs were taken from the commonly recommended sites for screening of colonisation, including hairline, nostrils, axilla, and hands $(26,27)$. Perineal swabs had to be excluded for reasons of compliance. A HCW was classified as a carrier if at least one of the swabs taken from four different sites tested positive for $S$. aureus.

\section{Microbiological methods}

Standard microbiological methods were followed to detect $S$. aureus among the staff members $(27,28)$. Swabs were first inoculated in salt nutrient broth. The broth was incubated at $37^{\circ} \mathrm{C}$ for 24 hours and then subcultured onto Mannitol salt agar (MSA). The plates were incubated at $35^{\circ} \mathrm{C}$ for 48 hours. Classification of colonies as $S$. aureus was verified using Gram staining and the coagulase test; $S$. aureus is Gram-positive and is distinguished from other staphylococci by its production of coagulase. The isolates were then tested for methicillin-resistance using oxacillin $(1 \mu \mathrm{g})$ discs on $2 \%$ salt Mueller Hilton agar incubated at $35^{\circ} \mathrm{C}$ for 24 hours (29); oxacilllin is similar to methicillin in terms of $\beta$-lactamase resistance and MRSA is able to grow in its presence whereas methicillin-sensitive $S$. aureus (MSSA) cannot. It should be noted, however, that testing positive for MRSA does not exclude the possibility that MSSA was also present at the test site.

\section{Patient Screening}

During the study period, 157 pus samples from patients admitted in the Burns Unit were processed in a manner similar to that described above in order to test for the presence of $S$. aureus. The number of samples yielding MRSA was noted. An attempt was also made to investigate the seriousness of the problem of MRSA carriage in the Burns Unit by studying the available surveillance reports and the number of patients infected or colonised with this organism.

\section{RESULTS}

Among the 34 hospital personnel screened in the Burns Unit, 26 (76\%) were found to be carriers of $S$. aureus (Table 1). Of these, 17 were carriers of MRSA (50\% of all HCWs) and 16 were carriers of MSSA, meaning that seven people tested positive for both MRSA and MSSA at different swab sites. As explained in the Methods, positive methicillin-resistance test results, while identifying MRSA, do not rule out the presence of MSSA. Rates of MSSA carriage may therefore be higher than estimated here based on cocolonisation of MSSA and MRSA at the same site.

Of the 26 people classified as carriers, 20 tested positive for $S$. aureus at more than one swab site. This suggests that the majority of carriers harbour the pathogen at multiple different sites on the body. Furthermore, only two people were classified as carriers based on a single positive result from the hands, therein bolstering the claim that $76 \%$ is a relatively accurate value for the rate of $S$. aureus carriage among HCWs in this ward (see Discussion). The finding of colonisation at more than one site is similar within the subpopulation of carriers with MRSA, where 13 of 17 people tested positive for MRSA at more than one site.

Table 2 documents at which of the four swab sites the $S$. aureus was being harboured. All four locations seemed to be common sites of $S$. aureus colonisation. In fact, $S$. aureus was most frequently found in the axilla, 
Table 1. Screening for S. aureus carrier status

\begin{tabular}{lcccc}
\hline Burns ward staff & No. of staff screened & No. positive MRSA & No. positive for MSSA & No. positive for both strains \\
\hline Doctors & 9 & 3 & 7 & 2 \\
Nurses & 22 & 12 & 8 & 4 \\
Nursing Assistants & 2 & 1 & 0 & 0 \\
Housekeeping staff & 1 & 1 & 1 & 1 \\
Total & $\mathbf{3 4}$ & $\mathbf{1 7}$ & $\mathbf{1 6}$ & $\mathbf{7}$ \\
\hline
\end{tabular}

a People included in this category are also counted in the other two categories, i.e. "No. positive for MRSA" and "No. positive for MSSA".

with $75 \%$ of all cariers being colonised at this site; the majority of colonisation at this site was, however, with MSSA. The nostrils were the second most common site of colonisation, with $71 \%$ of all carriers testing positive at this site; in this case, MRSA was 2.4 times more common than MSSA indicating that the nose is indeed a major reservoir of MRSA. Similarly, the hands also showed colonization predominantly by MRSA. Again, those sites testing positive for MRSA may also contain MSSA.

During the study period, microbiological culture reports revealed that of the 157 samples of pus processed from patients in the Burns Unit, 41 (21\%) yielded MRSA.

\section{DISCUSSION}

It is difficult to pin point the definite source of infection of MRSA in any outbreak situation. However, various studies have postulated that MRSA carriage by HCWs contributes to the occurrence of MRSA infection in clinical situations. The problem is compounded in the Burns Unit as patients are severely immunocompromised and receive numerous antibiotics. Care of these patients is often very labour-intensive, requiring many hours of hands-on contact (17).

At SJMCH, there is also no well defined protocol for the prevention and control of MRSA infection within the hospital as a whole, and staff often follow procedures specific to each unit. Furthermore, routine surveillance for staff carriage is not carried out. Prohibitive costs are often quoted as a deterrent to continuous surveillance for carriers among HCWs and screening of all patients admitted to high-risk areas. Moreover, the cost-effectiveness of screening staff has been questioned given that, in one study, relaxing such measures had little effect on the prevalence of MRSA (30). Attempts have been ongoing in this hospital to control the spread of nosocomial infection by awareness building, intensified hand washing, and isolation of patients, wherever possible. However the precise role of carriers in the spread of MRSA has not been studied. Continuous, ongoing education of all categories of staff is necessary to reiterate the fact that carriers could be a significant source of infection, especially for patient population such as that in the Burns Unit.

In this study a staggering $50 \%$ of the HCWs (17 out of 34 tested) working in this high-risk area were found to be carriers of MRSA. Similar figures were reported by Opal et al. (31) who found high rates (56\%) of staphylococcal colonisation among nurses $(65 \%$ of which were methicillin resistant) in an institution with chronic endemic MRSA. Crossley et al., however, in their study of a hospital outbreak involving burns patients (7) found that only $0.8 \%$ of $\mathrm{HCWs}$ were colonised with MRSA. The role of staff carriage in the spread of MRSA infection is controversial and the value of screening staff has been questioned by many authors $(20,30,32)$. In SJMCH, patients are initially admitted to single isolation rooms, but are cared for by the same team of HCWs. Attempts are being made to post colonised HCWs to low priority areas until MRSA eradication can be achieved.

The site most commonly colonised with MRSA among HCWs in this study was the anterior nares, followed by the hands. The anterior nares have also been identified as one of the most common sites of carriage in other studies (33) and, in fact, nasal carriage has been shown to increase the risk of wound infection (34). It is difficult to assess if colonisation of the hands is only transient carriage as no follow up of these carriers has been done in this study. However, of the ten HCWs colonised with MRSA on the hands, all but two had the organism at one or more other swab sites. This observation argues that few people could have been misclassified as carriers based on a spurious false positive result from one swab site. Mupirocin for nasal decolonisation is not readily available in India. Various other antiseptics such as chlorhexidine and neomycin have been tried. The outcome of these attempts however, is beyond the scope of this study.

It is difficult to assess the significance of isolation of a pathogen from a swab taken from a burn wound without doing semi-quantitative studies, as most wounds get colonised with potential pathogens (35). In this study, although MRSA was isolated from $21 \%$ of patients, no attempt was made to distinguish between colonisation and infection. Serial cultures and long term follow-up are required to assess the clinical outcome of 
Table 2. Sites of carriage of MRSA/MSSA

\begin{tabular}{lccc}
\hline Sites sampled $^{\mathbf{a}}$ & No. positive for S. aureus & No. positive for MRSA & No. positive for MSSA only ${ }^{\mathbf{b}}$ \\
\hline Hairline & 15 & $8(53 \%)$ & $7(47 \%)$ \\
Nostrils & 17 & $12(71 \%)$ & $5(29 \%)$ \\
Axilla & 18 & $7(39 \%)$ & $11(61 \%)$ \\
Hands & 15 & $10(67 \%)$ & $5(33 \%)$ \\
\hline
\end{tabular}

a Total number of sites sampled $=4 \times 36=136$

b MSSA could also be present in sites testing positive for MRSA

MRSA: Methicillin-resistant S. aureus; MSSA: Methicillin-sensitive S. aureus

colonisation/infection with this organism. No definite conclusions could be drawn with regard to the role of colonised HCWs contributing to infection of patients, since epidemiological marker studies were not done on any of these isolates of MRSA.

Prevention of MRSA infection merits discussion as, once introduced in a hospital, MRSA spreads widely among high-risk patients. The persistence of MRSA colonisation and the transfer of patients between wards/hospitals contribute to a constant reintroduction of MRSA into the institution. This pattern results in an ever-increasing reservoir of MRSA in many special care units, which in turn makes it difficult to control the spread of the organism. A number of infection control protocols have been formulated by various workers to be utilised for the eradication of MRSA colonisation from health care facilities $(12,36)$. None of these have succeeded in eradicating this pathogen from the hospital setting and some health care professionals have capitulated to MRSA and now accept it as a normal member of the hospital flora. There are however compelling reasons for the control of MRSA. Vancomycin is the primary antimicrobial agent used to treat MRSA infections. This drug is beyond the reach of the majority of patients with MRSA infections in India. Since an estimated $30-60 \%$ of patients colonised with this organism will become infected (12), the absence of affordable treatment is a very significant problem. Even when available, the emergence of resistance to vancomycin in $S$. aureus, as has been reported in Japan $(37,38)$, would pose a new threat to the control of nosocomial infections caused by this adaptable pathogen. Thus, the scarcity of treatment options and the morbidity and mortality associated with MRSA infection provide a strong argument for implementing strict rules to control the spread of this deadly pathogen within a hospital setting.

\section{ACKNOWLEDGEMENTS}

We wish to thank the staff of the Burns unit of St. John's Medical College Hospital, Bangalore, India, for their cooperation during this study. We also thank the technical staff of the Department of Microbiology, St.
John's Medical College for their assistance during this study.

\section{REFERENCES}

1. Lowy FD. Staphylococcus aureus infections. New England Journal of Medicine 339: 520-532; 1998.

2. Archer GL. Staphylococcus aureus: a well-armed pathogen. Clinical Infectious Diseases 26: 1179-1181; 1998.

3. Brumfit W, Hamilton-Miller J. Methicillin-resistant Staphylococcus aureus. New England Journal of Medicine 320: 11881196; 1989.

4. Barber M. Methicillin-resistant Staphylococcus aureus. Journal of Clinical Pathology 14: 385-393; 1961.

5. Jevons MP. 'Celbenin'-resistant Staphylococci. British Medical Journal 1: $124-125 ; 1961$.

6. Colley EW, McNicol MW, Bracken PM. Methicillin-resistant Staphylococcus aureus in a general hospital. Lancet 1: 595-597; 1965.

7. Crossley K, Landesman B, Zaske D. An outbreak of infections caused by strains of Staphylococcus aureus resistant to methicillin and aminoglycosides. Journal of Infectious Diseases 139: 280-287; 1979.

8. Fluckiger U. Epidemiology of methicillin-resistant Staphylococcus aureus. Chemotherapy 45: 121-134; 1979.

9. Cars O. Colonisation and infection with resistant gram-positive cocci. Epidemiology and risk factors. Drugs 54 (Suppl 6): 4-10; 1997.

10. Michel M, Gutman L. Methicillin-resistant Staphylococcus aureus and vancomycin-resistant enterococci: therapeutic realities and possibilities. Lancet 349: 1901-1906; 1997.

11. Ayliffe GA. The progressive intercontinental spread of methicillin-resistant Staphylococcus aureus. Clinical Infectious Diseases 24 (Suppl 1): S74-79; 1997.

12. Herwalt AH. Control of Methicillin resistant Staphylococcus aureus in the hospital setting. American Journal of Medicine 106: 11S-18S; 1999

13. Voss A, Milatovic D, Wallrauch-Schwarz C, et al. Methicillinresistant Staphylococcus aureus in Europe. European Journal of Clinical Microbiology and Infectious Diseases 13: 50-55; 1994.

14. Mehta A, Rodrigues C, Kumar R, et al. A pilot programme of MRSA surveillance in India. Journal of Postgraduate Medicine 42: 1-3; 1996.

15. John TJ. Emerging and re-emerging bacterial pathogens in India. Indian Journal of Medical Research 103: 4-18; 1996.

16. Mathur SK, Singhal S, Prasad KN, et al. Prevalence of methicillin-resistant Staphylococcus aureus in a tertiary care hospital in India. Indian Journal of Medical Microbiology 12: 96-101; 1994.

17. Hunt JL, Purdue GF, Tuggle DW. Morbidity and mortality of an epidemic pathogen: methicillin-resistant Staphylococcus aureus. 
American Journal of Surgery 156: 524-528; 1988.

18. Cook N. Methicillin-resistant Staphylococcus aureus versus the burn patient. Burns 24: 91-98; 1998.

19. Pruitt BA Jr, McManus AT, Kim SH, Goodwin CW. Burn wound infections: current status. World Journal of Surgery 22: 135-145; 1998.

20. Mulligan ME, Murray-Leisure KA, Ribner BS, et al. Methicillin-resistant Staphylococcus aureus: a consensus review of the microbiology, pathogenesis, epidemiology with implications for prevention and management. American Journal of Medicine 94: 313-325; 1993.

21. Perl TM, Golub JE. New approaches to reduce Staphylococcus aureus nosocomial infection rates: treating $S$. aureus nasal carriage. Annals of Pharmacotherapy 32: S7-16; 1998.

22. Muder RR, Brennen C, Wagener MM, et al. Staphylococcal colonization and infection in a long-term care facility. Annals of Internal Medicine 114: 107-112; 1991.

23. Monnet DL. Methicillin-resistant Staphylococcus aureus and its relationship to antimicrobial use: possible implications for control. Infection Control and Hospital Epidemiology 19: 552559; 1998.

24. Herwaldt LA. Control of methicillin-resistant Staphylococcus aureus in the hospital setting. American Journal of Medicine 106: 11S-18S; 1999.

25. Krishnan PU. Emergence of methicillin-resistance in Staphylococcus aureus in a medical college hospital. Abstracted in the Proceedings of First Indian Conference on Hospital Infection p. 77; 1992.

26. Duckworth GJ. Diagnosis and management of methicillinresistant Staphylococcus aureus. British Medical Journal 307: 1049-1052; 1993.

27. Mehtar S. Outbreaks Part 6. In: Hospital Infection Control, 1st Edition. . Oxford: Oxford University Press; 1992.

28. Shanson. Hospital Infection. In: Microbiology in clinical practice, 2nd Edition. London: Butterworth and Co; 1989.
29. National Committee for Control of Laboratory Standards (NCCLS). Performance standards for antimicrobial disk susceptibility test. Edition 4, M2-A4. Villanova, PA: NCCLS; 1991.

30. Crowcroft N, Maguire H, Fleming M, et al. Methicillin-resistant Staphylococcus aureus: investigation of a hospital outbreak using a case-control study. Journal of hospital infections 34: 301-309; 1996.

31. Opal SM, Mayer KH, Stenberg MJ, et al. Frequent acquisition of multiple strains of methicillin-resistant Staphylococcus aureus by health care workers in an endemic hospital environment. Infection Control and Hospital Epidemiology 11: 479-485; 1990.

32. Sheppard MJ. Control of methicillin-resistant Staphylococcus aureus. Journal of Hospital Infection 32: 73-78; 1996.

33. Locksley RM, Cohen ML, Quinn TC, et al. Multiply antibioticresistant Staphylococcus aureus: introduction, transmission, and evolution of nosocomial infection. Annals of Internal Medicine 97: 317-334, 1982.

34. Casewell MW. The nose: an underestimated source of Staphylococcus aureus causing wound infection. Journal of Hospital Infection 40 (Suppl): S3-11; 1998.

35. Lowbury EJ. Burn infection studies. Journal of hospital infection 32: 167-173; 1996.

36. Working Party of the Hospital Infection Society and British Society for Antimicrobial Chemotherapy. Revised guidelines for the control of epidemic methicillin-resistant Staphylococcus aureus. Journal of Hospital Infection 16: 351-377; 1990.

37. Hiramatsu K, Aritaka N, Hanaki H, et al. Dissemination in Japanese hospitals of strains of Staphylococcus aureus heterogeneously resistant to vancomycin. Lancet 350: 1670$1673 ; 1997$.

38. Casewell MW. New threats to the control of methicillin-resistant Staphylococcus aureus. Journal of Hospital Infection 30 (Suppl): 465-471; 1995.

Dr. Preetha Aravind is presenty doing her internship after completing an MBBS from Bangalore University at St. John's Medical College Hospital. Dr. Prabha Unny Krishnan, MBBS, MD, DNB is an Associate Professor in the Dept. of Microbiology; Dr. Hiresave Srinivasa, MBBS, MD holds the post of Professor in the Dept. of Microbiology; and Dr. Vijay Joseph, MBBS, MS, MCH is an Associate Professor in the Dept. of Plastic Surgery, all from St. John's Medical College Hospital, Bangalore, India. 